\title{
Swarm-driven idea models - from insect nests to modern architecture
}

\author{
S. von Mammen \& C. Jacob \\ Department of Computer Science, University of Calgary, Canada
}

\begin{abstract}
Inspired by the construction behavior of social insects we have developed a computational model of a swarm to build architectural idea models in virtual three-dimensional space. Instead of following a blueprint for construction, the individuals of a swarm react to their local environment according to an innate behavioral script. Through the interplay of the swarm with its environment, interaction sequences take place that give rise to complex emergent constructions. The configuration of the swarm determines the character of the emerging architectural models. We breed swarms that meet our expectations through artificial evolution, a very general framework for computationally phrased optimization problems. We present several evolved, swarm-built architectural idea models and discuss their potential in regards to modern ecological architecture.

Keywords: swarm grammar, ideal model, ecological architecture, bio-inspired computing, computer-aided design, computer-aided architectural design, generative representation, swarm intelligence, collective intelligence, artificial intelligence, artificial life, evolutionary computation, stigmergy, collaborative algorithm.
\end{abstract}

\section{Introduction}

Flocks of birds, schools of fish and bee hives are prominent examples of swarm systems. In these, large numbers of individuals interact by following simple stimulus-reaction rules. Globally, the swarm exhibits collectively intelligent behavior. Examples of their emergent abilities are nest constructions of social insect swarms, like termite mounds, wasp nests and ant galleries [1]. 
Artificial models of constructive insect swarms have successfully reproduced diverse nest architectures in computational simulations [2,3]. Hereby, local neighborhood information consisting of flock mates and environmental stimuli trigger the individuals' behaviors. Step-by-step intricate architectural solutions emerge by transporting construction elements and placing pheromones, while the individuals are unaware of the blue-print that appears in the eye of the observer.

We utilize computational swarm models for constructions in virtual 3D spaces [4-6]. The emerging constructions serve as idea models for architectural designs [7]. The behavioral patterns of different swarms lead to different outcomes. In order to obtain constructions that satisfy the expectations of the architect, swarms are bred through evolutionary algorithms [8]: First, the configurations of a population of swarms are randomly chosen and their constructional activities are computed. Different evaluation criteria are applied to rate the swarm constructions. Finally, a new population is generated based on a rating-dependent selection of previously evaluated swarms. Random changes (mutations) and recombinations of successful swarms give rise to new constructions. The repetition of this process yields diverse constructional designs. Most importantly, the evaluation criteria have to be formulated with great diligence to foster the emergence of interesting, yet well-constrained architectures. We found that a combined effort to direct the coordination of a swarm and to motivate construction within pre-defined shapes yields innovative results.

Although the presented architectural idea models are non-human products, they inherit the liveliness - in the sense of organic looks - adaptability, innovation and complexity of the virtual swarms that built them. Each of these features bears the potential to contribute to the design of modern eco-architecture.

For the rest of this article we retain the sequence of topics as in this introduction, starting with a brief introduction to construction techniques of social insects, followed by an overview of the applied computational concepts. After the presentation and characterization of several swarm-driven architectural idea models, their features are discussed and further opportunities for swarm-based architectural design are explored.

\section{The architecture of social insects}

Only about 3 to $5 \%$ of all animal species have evolved from solitary to social organizations [9]. Among them are humans as well as social insects, such as ants or wasps. Due to the synergy of collectives, this small percentage has been having a major impact on life. This is also reflected in the homes of social earth-dwellers: They enjoy architectures of ingenuity, complexity and (relative) size unsurpassed by any solitary species. Our approach to production deviates dramatically from theirs [1]. We diligently engineer plans to reach our goals, whereas ants seem to follow scripts that determine simple stimulus-reaction behaviors $[2,10]$. On the one hand, social insects are unaware of their works' contribution to the greater good. On the other hand, they naturally construct intricate nests. They construct efficiently, are well coordinated and utilize the massive power of large 
numbers of individuals that build in parallel $[11,12]$. Insect nests primarily serve reproduction, i.e. protection and breeding, but they also realize social functions swarm cohesion, identity and communication [13]. For predator defense they are equipped with chemical repellents and have evolved construction features such as envelopes and strategic entrance points [14,15]. Nests can be built despite the impact of harsh natural forces, such as wind [16]. They offer shelter against temperature fluctuations through overlapping and insulating envelopes [13], or through ventilation systems that interconnect chimneys and chambers of varying exothermic properties [1].

The community effort of nest construction happens in several phases. In each phase a certain subshape is built which in turn determines a module for the next phase. Nest construction could actually be realized solely through manipulation and interpretation of the built constructions, which is coined sematectonic communication. It is important that the construction phases are clear and no sematectonic cues overlap [2,11-13]. This would trigger uncoordinated behaviors in the individuals, leading to incoherent buildings. Additionally, communication among insects happens through the placement and smell of pheromones. Stigmergy refers to the communication through the environment in general and thereby subsumes sematectonic and pheromone-driven communication. Stigmergy comes in two flavors (1) qualitative, where a specific pheromone or construction triggers a behavior, and (2) quantitative, where the intensity of a stimulus correlates with the triggered activities.

Social insects follow a decentralized, self-organizing construction approach whose effectiveness and efficiency inspires us to learn from their technologies. In the next section, we present a concept of an artificial swarm to develop novel architectural designs.

\section{Artificial life or how to evolve a swarm?}

Assumptions about the ways of collaboration of social insects are often based on computational simulations $[2,3,16]$. The results are carefully termed 'assumptions' as any computational model is a simplification of nature. It is the responsibility of the scientist to decide, how the insects are represented and how they can interact in the computer simulation. If phenomena emerge numerically that are also seen in nature, we can only assume that the underlying computational model matches the actual objective.

In 1987, Reynolds presented a computational model of flocking birds, or 'boids' [17]. Three-dimensional cones that flew through a virtual space were driven to align with their flock-mates, to keep a minimal distance and to urge towards the center of their perceived neighbors. Many other models that are inspired by living systems have been developed, for instance motivated by the growth of bacterial cultures and plants [18-20]. These biologically inspired models can confirm scientific hypotheses (e.g. about the semitectonic communication of wasps), but they can also utilize the innovative mechanisms found in nature to solve other challenges. 


\subsection{Self-organized, bio-inspired architecture}

With the goal to create self-organized bio-inspired architecture, we have designed a model of an artificial swarm that flocks coherently like Reynolds' boids [17], that communicates and constructs like social insects [2] and whose individuals can differentiate like prokaryotic cells and reproduce like bacteria [20]. Combining these three modeling technologies results in a new computational concept called swarm grammars [5,6]. In particular, a swarm grammar consists of several individuals that perceive and react to their local environments. On the one hand, the individuals' flight is regulated by several parameters, the so-called flocking parameters. On the other hand, a set of behavioral rules determines which further actions should be taken under certain conditions. For instance, upon perception of a stigmergic stimulus, such as a specific construction shape, a swarm individual might place a construction element. Thus, constructions can grow in three-dimensional space during the swarm simulations. An activity, such as building, is not necessarily triggered by a stigmergic stimulus. An internal timer or mere chance could also determine an individual's behavior. At the same time, building is not the only action the individuals can perform. They could also reproduce themselves, die out, or differentiate into individuals with different flight parameters and behavioral rules.

The number of possible interactions between swarm individuals, and hence the number of possible swarm grammar constructions is vast. The question arises how to configure a swarm grammar that grows innovative architectural idea models. The configuration of a swarm grammar does not reveal the corresponding emerging construction right away. Only the simulation of a swarm grammar sheds light on its emergent productive achievements, since the swarm interactions and the resulting building processes are highly non-linear.

\subsection{Evolving swarm architecture}

The duality of configuration and outcome can be easily translated into biological terms: Swarm grammar configurations represent genotypes and their simulations compute the corresponding architectures, the phenotypes. Rephrasing our task, we need to find genotypes that produce fit phenotypes. In order to breed phenotypes that meet our expectations we utilize some of the concepts of evolution [8]. First, the fitnesses of phenotypes of randomly configured swarm grammars are measured (Simple examples of fitness measures are: symmetry, size, or structural rigidity.). The assigned fitness values determine the probability of a genotype's selection for reproduction into the next generation. During the reproduction process, the selected genotypes recombine or mutate, possibly producing fitter swarm grammars for the next generation. After the computation of a number of generations, we can expect that the average fitness has risen considerably. Recombination basically means that parts of a genotype $A$ are exchanged with the corresponding parts of a second genotype $B$. In the given problem, randomly chosen flocking parameters and behavioral rules are exchanged. Mutation directly operates on parts of the genotype, its genes. For instance, a behavioral rule could change from 'If 
a rod construction element is perceived, build a cube construction element' into 'If a rod construction element is perceived, build a layer construction element', whereas the flocking parameter to weigh an individual's alignment urge could change from 0.78 to 0.73 . The selection process, recombination and mutation are well-established steps in a genetic algorithm, whereas the formulation of a fitness criterion to evaluate the phenotypes can be a difficult task.

In order to promote those phenotypes that meet our expectations of architectural idea models, we foster the construction within pre-defined boundaries [4], and we measure indications of the emerging complexity of the swarm-built architectures. In particular, we measure the heterogeneity of the swarms during the construction process, e.g. the number of different swarm individuals, and the level of swarm collaboration, e.g. the average number of swarm neighbors and swarm interactions. Uncontrolled reproduction of swarm grammar individuals could quickly lead to an exponentially growing demand for computing resources. In order to avoid such an excess of resource usage, a simulation process taking longer than 100 real seconds is terminated and is not considered for further evolution. These measurements for effective fitness assignment can be summarized as: (1) promotion of construction within pre-defined boundaries, (2) promotion of heterogeneity and collaboration, and (3) prevention of constructional outgrowth. These measures are summed up in the fitness value of a phenotype. The fitness assignment concludes the genetic algorithm which now can produce swarm grammar configurations that create architectural idea models.

\section{Ecological swarm architecture}

From a wide variety of designs, a selection of four architectural idea models is presented in the following paragraphs. Based on the given examples, we discuss the potential of the approach of artificially evolving swarm-built architectures in regards to ecological architecture.

\subsection{Idea models created by artificial swarms}

Figure 1 depicts an architectural idea model that is composed by several interwoven ripples of layer constructions. A homogeneous swarm of five individuals swirls around a declining path while dropping layers and rods. At the bottom (Fig.1(a)), two flocks are gaining distance, thereby splitting the construction. During the evolutionary process the genotype of the given example received credit for the number of swarm interactions and for the good size of the construction. The skeletal structure of Figure 2 is assembled of rods and cubic construction elements. Several swarm individuals wrap around and cement an inner construction with waves of rods. Crucial for this interplay is a probability-driven reproduction of the 'foremen' and their differentiation into mere operative swarm individuals that do nothing but place construction elements. The emergence of a tight flocking pattern also strongly influenced this outcome. Figure 3 displays a very complex swarm grammar phenotype: During the construction process individuals spawn several 


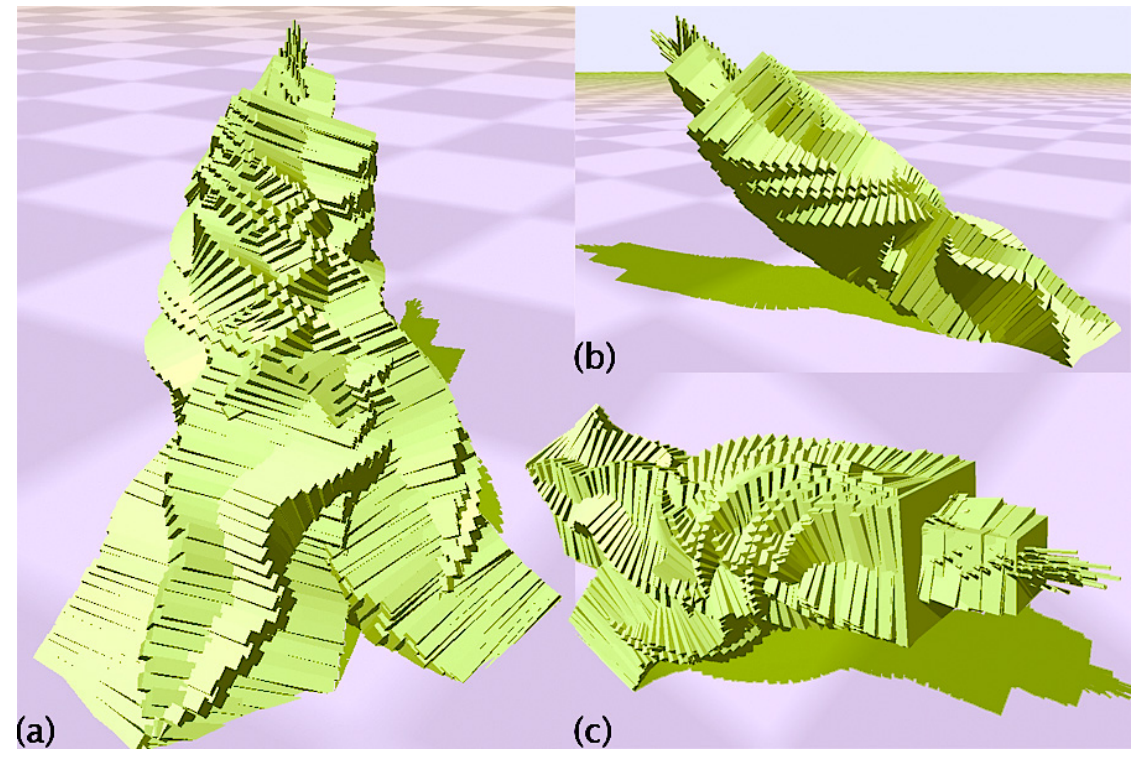

Figure 1: A swarm architecture from different perspectives: (a) front view, (b) side view, (c) top view.

hundred times which leads to long computation times. During the interplay of the expressed swarm individuals, one of them is responsible for the reproduction and differentiation. One individual only places rods, another one only layers. A fourth involved individual places a rod, a cube and a layer construction element all at once but with a very low probability. Figure 4 grows an extended set of rods and cubic construction elements on top of the bottom-up sequence of layers.

\subsection{Nature-inspired, organic, adaptive, diverse design}

The models in Figures 1 to 4 demonstrate how biological construction processes can be adopted to create nature inspired architectural models [21,22]. As we will explain in the following paragraphs, the outlined bio-inspired approach inherently promotes organic aesthetics, produces individual solutions for specific environments and offers dynamic and diverse designs.

The predominance of rectangular, cubic elements, the emphasize of 'the straight line' is a relic of the industrial revolution and not desirable. Instead, an organic style with 'free-flowing curves' should be favored which is supported by modern construction processes and materials [23]. Numerous local interactions of swarm grammar individuals realize this aesthetic demand. Figures 1 to 3 bristle with round shapes, ripples of construction elements and harmonically interwoven structures. Figure 4 depicts the most rigid of the four presented models. Terraces at the bottom level, in the middle and at the top (Figs. 4(b) and (c)) that are connected through cascading rectangular layers lend the model a systematic structure. But 

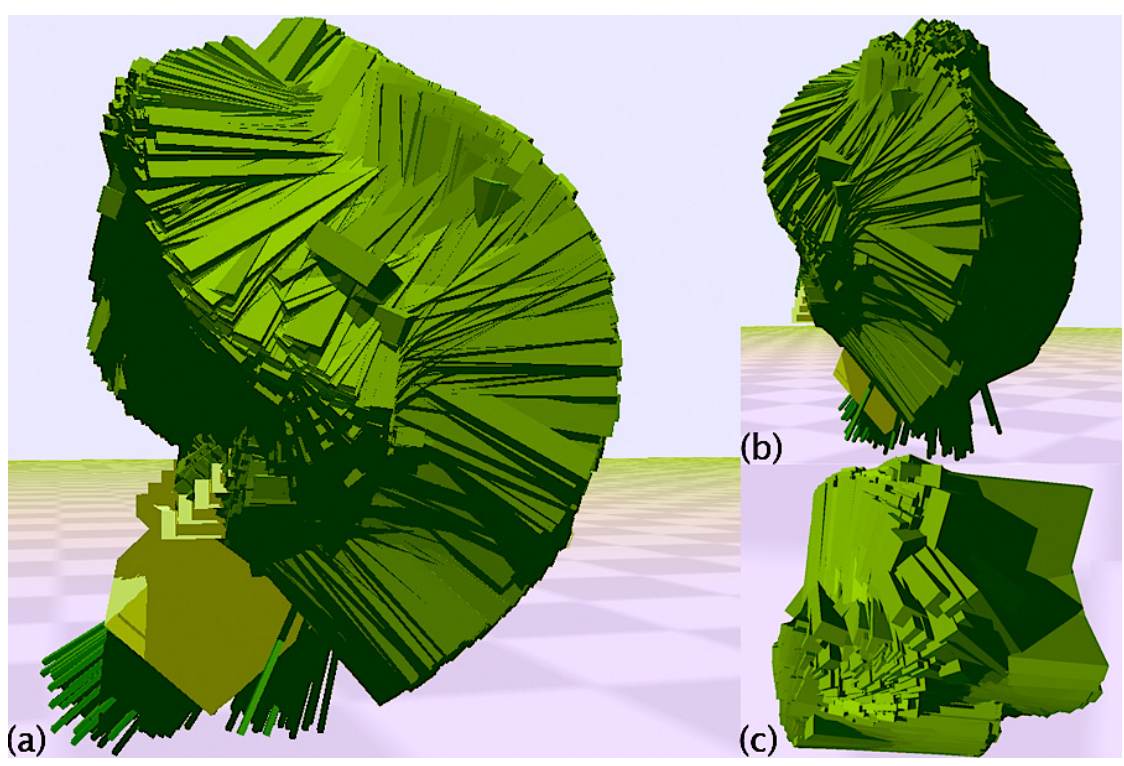

Figure 2: A swarm architecture from different perspectives: (a) side view (b) front view, (c) top view.

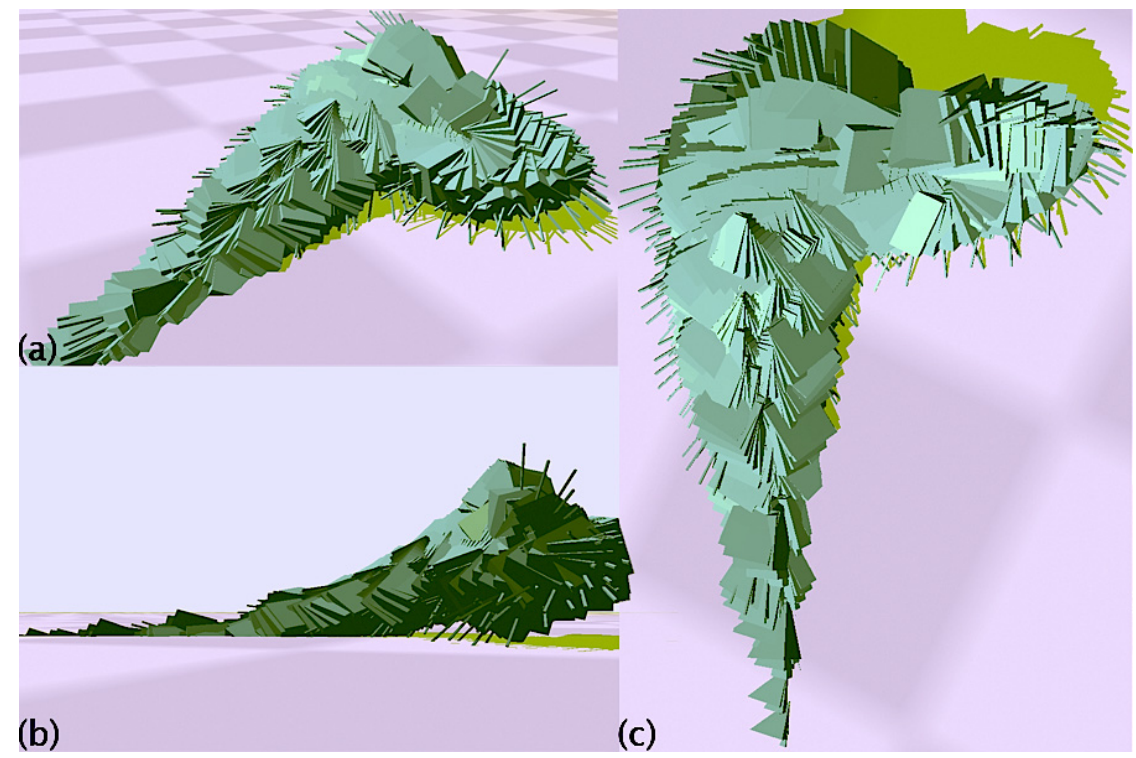

Figure 3: A swarm architecture from different perspectives: (a) from a $45^{\circ}$ angle, (b) side view, (c) top view. 


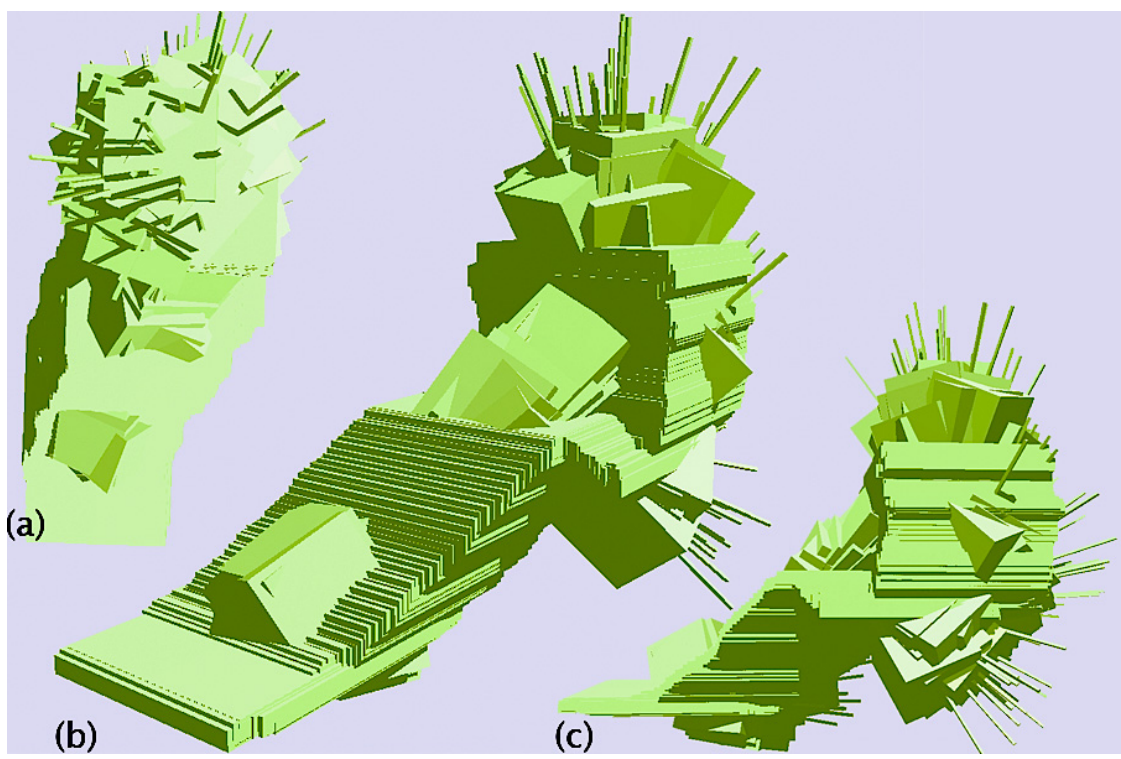

Figure 4: A swarm architecture from different perspectives: (a) top view, (b) from a $45^{\circ}$ angle, (c) side view.

even in this example the flow of spontaneously placed construction elements, protruding cubes and rods convey an organic, flowing character of the architecture.

Modern architecture needs to be integrated into the environment, the 'sitespecific context' has to be taken into account [21]. The swarm-driven construction approach responds to this requirement on two levels. On the one hand, swarm individuals are aware of their environment and act accordingly. Through this stigmergic mechanism the constructions in Figures 1 to 4 emerged. On the other hand, artificial evolution of constructive swarms can be utilized to optimize the constructions in regards to waste water disposal, energy efficiency and other aspects of ecological and economic performance [24]. Therefore, it is necessary to integrate supplementary software modules that evaluate these features of performance [25]. In addition to swarm heterogeneity and collaboration, ecological performance would direct the evolution of swarm-built architectures.

\section{Summary and future work}

We have developed a computational swarm model which incorporates flight coordination of natural swarms, growth and reproduction abilities of plants and bacteria, and the construction methodology of social insects. By means of artificial evolution we have bred swarms that construct innovative organic architectures in virtual space. Based on several examples, we argue that swarm-driven architecture naturally fulfills ecological principles such as adoption of a biological construction mechanism, organic aesthetics, diversity and environmental adaptation. The next 
step in the evolution of swarm-driven architecture could be the promotion of features of ecological efficiency.

\section{References}

[1] Hölldobler, B. \& Wilson, E.O., The Ants. Springer-Verlag: BerlinHeidelberg, 1990.

[2] Bonabeau, E., Dorigo, M. \& Theraulaz, G., Swarm Intelligence: From Natural to Artificial Systems. Oxford University Press: New York, 1999.

[3] Pilat, M., Wasp-inspired construction algorithms. Technical report, University of Calgary, 2004.

[4] von Mammen, S., Jacob, C. \& Kokai, G., Evolving swarms that build 3d structures. 2005 IEEE Congress on Evolutionary Computation, volume 2, pp. 1434-1441 Vol. 2, 2005.

[5] Jacob, C. \& von Mammen, S., Swarm grammars: growing dynamic structures in 3D agent spaces. Digital Creativity: Special issue on Computational Models of Creativity in the Arts, 18, 2007.

[6] von Mammen, S. \& Jacob, C., Genetic swarm grammar programming: Ecological breeding like a gardener. 2007 IEEE Congress on Evolutionary Computation, eds. D. Srinivasan \& L. Wang, IEEE Computational Intelligence Society: Singapore, IEEE Press, pp. 851-858, 2007.

[7] Knoll, W. \& Hechinger, M., Architektur-Modelle: Anregungen zu Ihrem Bau. Deutsche Verlangs-Anstalt: Munich, Germany, 2006.

[8] Jacob, C., Illustrating Evolutionary Computation with Mathematica. Morgan Kaufmann: San Francisco, 2001.

[9] Thaler, W. \& Hölldobler, B., Ants - nature's secret power. Documentary Film. ORF - Natural History Unit. Vienna, Austria, 2004.

[10] Karsai, I. \& Penzes, Z., Comb building in social wasps: Self-organization and stigmergic script. Journal of Theoretical Biology, 161(4), pp. 505-525, 1993.

[11] Theraulaz, G. \& Bonabeau, E., Modelling the collective building of complex architectures in social insects with lattice swarms. Journal of Theoretical Biology, 177(4), pp. 381-400, 1995.

[12] Theraulaz, G. \& Bonabeau, E., Coordination in Distributed Building. Science, 269(5224), pp. 686-688, 1995.

[13] Camazine, S.e.a., Self-Organization in Biological Systems. Princeton University Press: Princeton, 2003.

[14] Jeanne, R., The Adaptiveness of Social Wasp Nest Architecture. The Quarterly Review of Biology, 50(3), pp. 267-287, 1975.

[15] Smith, A., O’Donnell, S. \& Jeanne, R., Correlated evolution of colony defence and social structure: A comparative analysis in eusocial wasps(Hymenoptera: Vespidae). Evolutionary Ecology Research, 3(3), pp. 331-344, 2001.

[16] Ladley, D. \& Bullock, S., Logistic constraints on 3d termite construction. the Fourth International Workshop on Ant Colony, eds. M. Dorigo, M. Birattari, L.M. Blum, F. Mondada \& T. Stutzle, Springer, Berlin, pp. 178-189, 2004.

[17] Reynolds, C.W., Flocks, herds, and schools: A distributed behavioral model. 
SIGGRAPH '87 Conference Proceedings, volume 4, pp. 25-34, 1987.

[18] Hoar, R., Penner, J. \& Jacob, C., Transcription and evolution of a virtual bacteria culture. IEEE Congress on Evolutionary Computation, IEEE Press: Canberra, Australia, 2003.

[19] Penner, J., Hoar, R. \& Jacob, C., Bacterial chemotaxis in silico. ACAL 2003, First Australian Conference on Artificial Life, Canberra, Australia, 2003.

[20] Prusinkiewicz, P. \& Lindenmayer, A., The Algorithmic Beauty of Plants. Springer-Verlag, 1996.

[21] Bergen, S., Bolton, S. \& L. Fridley, J., Design principles for ecological engineering. Ecological Engineering, 18(2), pp. 201-210, 2001.

[22] Van der Ryn, S. \& Cowan, S., Ecological Design. Island Press, 2007.

[23] Pearson, D., New Organic Architecture: The Breaking Wave. University of California Press, 2001.

[24] Anselm, A.J., Developing designs in balance with nature. Eco-Architecture: Harmonisation between Architecture and Nature, eds. G. Broadbent \& C. Brebbia, Wessex Institute of Technology, WIT Press, Transactions on the Built Environment, pp. 195-204, 2006.

[25] Gowri, K., Green building rating systems: An overview. ASHRAE Journal, 46(11), pp. 56-60, 20041101. 